OPEN ACCESS

Edited by:

Peter A. Federolf,

University of Innsbruck, Austria

Reviewed by:

Richard DeWeese,

Civil Aerospace Medical Institute,

United States

Wolfgang Immanuel Schöllhorn,

Johannes Gutenberg University

Mainz, Germany

${ }^{*}$ Correspondence:

Rud Derie

rud.derie@ugent.be

Pieter Robberechts

pieter.robberechts@cs.kuleuven.be

Specialty section:

This article was submitted to

Biomechanics,

a section of the journa

Frontiers in Bioengineering and

Biotechnology

Received: 31 October 2019

Accepted: 15 January 2020

Published: 04 February 2020

Citation:

Derie $R$, Robberechts $P$ Van den Berghe P, Gerlo J, De Clerca D, Segers V and Davis J (2020) Tibial Acceleration-Based Prediction of Maximal Vertical Loading Rate During Overground Running: A Machine Learning Approach. Front. Bioeng. Biotechnol. 8:33. doi: 10.3389/fbioe.2020.00033

\section{Tibial Acceleration-Based Prediction of Maximal Vertical Loading Rate During Overground Running: A Machine Learning Approach}

\author{
Rud Derie ${ }^{1 *}$, Pieter Robberechts ${ }^{2 *}$, Pieter Van den Berghe ${ }^{1}$, Joeri Gerlo', \\ Dirk De Clercq ${ }^{1}$, Veerle Segers ${ }^{1}$ and Jesse Davis ${ }^{2}$
}

\begin{abstract}
'Department of Movement and Sports Sciences, Ghent University, Ghent, Belgium, ${ }^{2}$ Department of Computer Science, KU Leuven, Leuven, Belgium
\end{abstract}

Ground reaction forces are often used by sport scientists and clinicians to analyze the mechanical risk-factors of running related injuries or athletic performance during a running analysis. An interesting ground reaction force-derived variable to track is the maximal vertical instantaneous loading rate (VILR). This impact characteristic is traditionally derived from a fixed force platform, but wearable inertial sensors nowadays might approximate its magnitude while running outside the lab. The time-discrete axial peak tibial acceleration (APTA) has been proposed as a good surrogate that can be measured using wearable accelerometers in the field. This paper explores the hypothesis that applying machine learning to time continuous data (generated from bilateral tri-axial shin mounted accelerometers) would result in a more accurate estimation of the VILR. Therefore, the purpose of this study was to evaluate the performance of accelerometer-based predictions of the VILR with various machine learning models trained on data of 93 rearfoot runners. A subject-dependent gradient boosted regression trees (XGB) model provided the most accurate estimates (mean absolute error: $5.39 \pm 2.04 \mathrm{BW} \cdot \mathrm{s}^{-1}$, mean absolute percentage error: $6.08 \%$ ). A similar subject-independent model had a mean absolute error of $12.41 \pm 7.90 \mathrm{BW} \cdot \mathrm{s}^{-1}$ (mean absolute percentage error: 11.09\%). All of our models had a stronger correlation with the VILR than the APTA $(p<0.01)$, indicating that multiple 3D acceleration features in a learning setting showed the highest accuracy in predicting the lab-based impact loading compared to APTA.

Keywords: running biomechanics, impact loading, tibial shock, machine learning, wearable sensor, gait analysis

\section{INTRODUCTION}

Ground reaction forces are relevant parameters for running analysis (Pohl et al., 2009; Crowell and Davis, 2011; Van Der Worp et al., 2016; Clark et al., 2017). They partially describe the center of mass' state of motion during running and are often used by sport scientists and clinicians to analyze the mechanical risk-factors of running related injuries (Bredeweg et al., 2013; Napier et al., 2018) and/or athletic performance (Preece et al., 2019). 
A commonly used ground reaction force-derived variable is the maximal vertical instantaneous loading rate (VILR), which is calculated as the maximal slope of the rising vertical ground reaction force - time curve (Ueda et al., 2016). VILR has been used to characterize the impact (i.e., high rate of force development due to the rapid deceleration of all body segments during the foot-ground collision) during running (Gerritsen et al., 1995). This measure could discriminate groups of rearfoot runners with a history of stress fractures (Van Der Worp et al., 2016) and plantar fasciitis (Pohl et al., 2009). Consequently, VILR has been considered clinically relevant and has been a main outcome variable in gait retraining studies targeting runners with high VILR (Crowell and Davis, 2011; Clansey et al., 2014; Willy et al., 2016).

Ground reaction forces are traditionally measured using fixed force platforms or instrumented treadmills (Ueda et al., 2016). Unfortunately, measurements with force platforms are laboratory-based and require both expensive equipment and extensive post-processing. These factors limit the potential of monitoring in-field running biomechanics, whereas wearable inertial measurement units can accommodate this by predicting running gait parameters outside the laboratory (Falbriard et al., 2018; Wouda et al., 2018). In this respect, an ambulatory low-cost accelerometer was proposed as a potential surrogate candidate to estimate VILR when force platforms are not available (Ngoh et al., 2018). Previous research has identified a moderate to good correlation (range of $r_{\text {mean }}=0.64-0.84$ ) between the axial peak tibial acceleration (APTA) captured by a skin-mounted accelerometer at the tibia and VILR (Laughton et al., 2003; Pohl et al., 2009; Greenhalgh et al., 2012; Zhang et al., 2016; Van den Berghe et al., 2019). Therefore, using APTA as a surrogate measure for VILR seems justifiable (Sheerin et al., 2019).

However, the APTA is based on a single, basic feature (i.e., the peak value) of the time-continuous $1 \mathrm{D}$ tibial acceleration signal. Consequently, a large amount of data is neglected, which may lead to missing important information. A combination of multiple features of the $3 \mathrm{D}$ tibial acceleration signals, possibly including complex and higher-order ones, may result in a more accurate predictor of VILR than only considering APTA. Hence, a performant computational model that extracts relevant features and effectively copes with any non-linear relationships (between the features of the tibial acceleration signals and the target VILR) is desired. In that way, machine learning techniques could help to analyze continuous time-series data without pre-selecting discrete variables. Holzreiter and Köhle (1993) introduced the use of neural networks to assess gait patterns in locomotion biomechanics. Recently more advanced machine learning techniques have been used to detect pathologic gait-patterns (Williams et al., 2015; Zeng et al., 2016), fatigue (Janssen et al., 2011; Op De Beéck et al., 2018) as well as classifying gender, performance-level (Clermont et al., 2018) and age-related running patterns (Fukuchi et al., 2011).

To gain a better understanding of the relationship between the external load and potential injury risk in overground running, a more accurate estimation of the athlete's impact loading is an essential methodological prerequisite. The screening of runners on impact intensity could be more accurate by estimating
VILR by means of a machine-learned model instead of relying on the APTA only. Consequently, this study proposes and evaluates the performance (e.g., predictive accuracy, calculation time, diagnostic ability) of an inertial sensor-based method to estimate the runner's VILR based on bilateral 3D shin-mounted accelerometer data using a machine learning approach. It was hypothesized that the incorporation of these extracted features into a set of machine-learned models would result in stronger predictive and diagnostic capacities than considering APTA only.

\section{MATERIALS AND METHODS}

\section{Ethics Statement and Participants}

Ninety three subjects engaged in recreational as well as competitive running (55 men and 38 women) were recruited from the local community. Runners were included if they were free of running-related injuries and ran at least $15 \mathrm{~km}$ per week (Table 1). All subjects signed an informed consent prior to the testing. Approval for the study was obtained from the ethical committee of the Ghent University Hospital (2015/0864).

\section{Protocol and Setup}

All runners were equipped with a backpack/tablet system to measure the tibial accelerations (Van den Berghe et al., 2019). Two tri-axial accelerometers (LIS331, Sparfkun, Colorado, United States; $1000 \mathrm{~Hz}$ /axis), were as tight as tolerable strapped with sports tape on the antero-medial side of both tibias, $8 \mathrm{~cm}$ above the malleolus medialis (Laughton et al., 2003; Clansey et al., 2014). The axis of each accelerometer was orientated in a way that the vertical axis of the accelerometer coincided with the longitudinal axis of the concerned tibia. The skin around the lower leg was pre-stretched with sports tape to improve the rigid coupling between the accelerometers and the tibia (Clansey et al., 2014; Van den Berghe et al., 2019). Data collection took place during two different projects, but with an exact same measurement setup.

The first cohort consisted of 13 subjects who were asked to run on a $30 \mathrm{~m}$ instrumented running track at multiple running speeds $\left(2.55 \mathrm{~ms}^{-1}, 3.20 \mathrm{~ms}^{-1}, 5.10 \mathrm{~ms}^{-1}\right.$, and preferred running speed). All subjects were habitual rearfoot strikers and were provided with the same standardized neutral distance running shoe (Li Ning Magne, ARHF041). The second cohort consisted of 80 runners running at $3.20 \mathrm{~m} \cdot \mathrm{s}^{-1}$. Subjects were not preselected on their habitual footstrike pattern and received no verbal instruction about the desired footfall pattern. They wore

TABLE 1 | Characteristics of the subjects.

\begin{tabular}{lccccc}
\hline & \multicolumn{2}{c}{ Men } & & \multicolumn{2}{c}{ Women } \\
\cline { 2 - 3 } & Mean & SD & & Mean & SD \\
\hline Age (Yrs.) & 35.9 & 9.2 & & 34.6 & 10.8 \\
Body height $(\mathrm{m})$ & 1.79 & 0.07 & & 1.67 & 0.06 \\
Body mass $(\mathrm{kg})$ & 76.5 & 10.2 & & 60.6 & 7.3 \\
Training volume $(\mathrm{km} /$ week $)$ & 36.4 & 16.9 & & 27.9 & 11.0
\end{tabular}




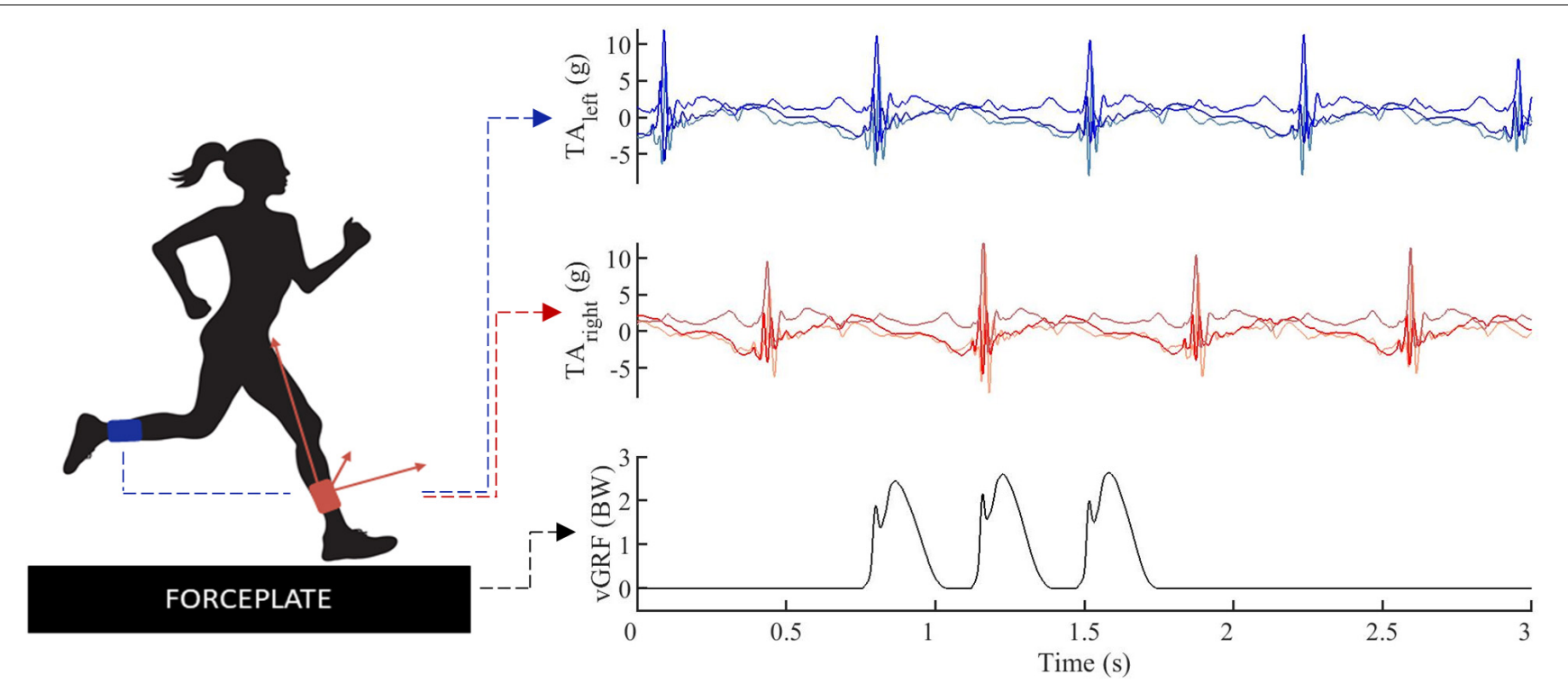

FIGURE 1 | Data example, tri-axial.1pc accelerations (TA) were simultaneously captured for the left (blue) and right (red) lower leg, vertical ground reaction forces (vGRF) were synchronized in time (black).

their regular training shoes. In both cohorts running speed was controlled by timing gates. Recorded trials were discarded and the runners received verbal feedback if their running speed was not within a $0.2 \mathrm{~m} \cdot \mathrm{s}^{-1}$ of the targeted speed. Ground reaction forces were measured at $1000 \mathrm{~Hz}$ by two built-in force platforms ( 2 and $1.2 \mathrm{~m}$, AMTI, Watertown, MA, United States). Accelerometer and force data were synchronized in time (Figure 1) by means of an infrared impulse sent from the motion capture system. The pulse was captured by an infrared sensor attached to the backpack system. For a more detailed description of this synchronization protocol we refer to Van den Berghe et al. (2019).

\section{Data Processing}

\section{Example Construction and Data Preprocessing}

Ground reaction force data were filtered using a zero-lag second-order low-pass Butterworth filter with a cutoff frequency of $60 \mathrm{~Hz}$. VILR was calculated as the maximal value of the first derivative of the vertical ground reaction force component following initial contact (vertical ground reaction forces exceeding a $5 \mathrm{~N}$ threshold) (Ueda et al., 2016). This was subsequently normalized to the subject's body weight. The acceleration signals were filtered in order to separate the linear acceleration from the gravity component and remove highfrequency noise using the approach of van Hees et al. (2013). The filtering settings were selected using a tuning procedure where $2 / 3$ of the data was used to train a model and $1 / 3$ to evaluate the model. First, to find a sensible range for the parameters, a manual exploration was performed using Chebyshev (type I and type II) and Butterworth filters with settings derived from related research. Subsequently, a grid search of Butterworth filters $[(0.2,1.0$; step $=0.2) \times(40.0,70.0$; step $=5)]$ was applied to the acceleration signals and the filter which resulted in the best performance on the evaluation set was selected, which was a second-order band-pass filter with cutoff frequencies of 0.8 and $45 \mathrm{~Hz}$ (Figure 2).

We extracted individual strides by splitting the collected signals at the take-off events of the opposite feet. This guarantees that each window contains the part of the acceleration signal that is relevant for determining the VILR. Next, we mirrored the data from the right and left leg, such that each of these strides starts with the right leg making ground contact. This procedure effectively doubled the amount of training data.

Each of the 93 subjects completed on average 16 trials (range: 6 to 67 trials), with each trial containing 2.67 strides on average. In total, 23 trials were removed from the data set due to clear errors in measured ground reaction forces and/or tibial accelerations. This resulted in 4037 examples in total.

\section{Feature Construction}

A large set of features consisting of three broad categories was considered: (1) auto-generated statistical features of the $3 \mathrm{D}$ acceleration waveforms, (2) trial-specific features, and (3) subject-describing features (Figure 2).

\section{Auto-generated statistical features}

First, from the tri-axial filtered acceleration signals of both feet, we extracted the window between the initial ground contact event and the event where the vertical acceleration component reaches $0 \mathrm{~g}$. Next, we calculated a comprehensive set of timeseries features from these windows using the TsFresh Python package (Christ et al., 2018). The extracted features include both basic characteristics of the signals (e.g., mean, maximum, number of peaks, timing of peak values) and more complex features (e.g., continuous wavelet coefficients, coefficients of an autoregressive model, the time reversal symmetry statistic, Fourier coefficients). We refer to the TsFresh paper (Christ et al., 2018) for a full description and list of features. 


\section{Data Preprocessing and Feature Engineering}



FIGURE 2 | Data preprocessing and feature engineering part of the machine learning pipeline. First, the raw acceleration signals were filtered using a Butterworth bandpass filter. The optimal filter configuration was determined by training multiple models, using different filter configurations. The configuration which enabled the most accurate predictions was used henceforth. Second, feature engineering was used to derive a lower-dimensional representation of the data. The generated features were a combination of automatically generated statistical features and manually crafted domain-specific features. The set of automatically generated features was reduced using a univariate feature selection technique.

The FRESH procedure (Christ et al., 2017) was used for feature selection. First, this procedure evaluates the influence of every feature on the target (VILR) using a univariate test (i.e., Kendall rank test for real-valued features and Kolmogorov-Smirnov for binary features) and computes the $p$-value. So, it tests whether the feature and the target are not statistically independent. Subsequently, the BenjaminiYekutieli procedure was carried out to control for the false discovery rate. This procedure reduced the set of auto-generated features to 1662 .

\section{Trial-specific features}

Running speed, derived from timing gates (Van den Berghe et al., 2019), and ground contact time, derived from tibial accelerations, were included as trial-specific features for each stride. Because the ground contact time cannot be inferred directly from the tibial acceleration signals, we modeled this as a separate prediction problem. Specifically, we solved the related task of predicting the timings of the initial contact and toe off gait events. The ground contact time can then be inferred from the time difference between both events. Due to the interrelations between both gait events (e.g., a toe off event follows 160 to $350 \mathrm{~ms}$ after an initial contact event), we framed this as a structured prediction task. In this framework, a function between the acceleration profile and a sequence of initial contact and toe off timings was learned. Specifically, a deep structured recurrent neural network architecture was used. The neural network component of the model used the raw acceleration signals, the jerk (first order derivative of acceleration signals), roll $\left(\arctan \left(a_{y} \cdot a_{z}\right)\right)$ and pitch $\left(\arctan \left(-a_{x} \sqrt{a_{y}^{2}+a_{z}^{2}}\right)\right)$ of both legs to infer the likelihood of a gait event happening for each sample. Subsequently, the structured component consisted of a constrained peak detection algorithm on the likelihood function that finds the most likely combination of initial contact and toe off timings. Both components were optimized jointly. For a detailed description of this model, see Robberechts et al. (2019).

\section{Subject-describing features}

Third, the body weight and the shoe type were included. The weight of each subject is a logical feature to consider since the loading rate is expressed as a function of body weight. Furthermore, earlier research has found that footwear properties may affect VILR, even with similar foot-strike patterns (Kulmala et al., 2018). When testing the second cohort $(n=80)$, the subjects reported their shoe brand and type. The shoe's properties were verified through online databases (running shoes guru, solereview, runner's world, manufacturer's website, etc.) and subsequently categorized as being neutral, stabilization or racing flats.

\section{Learning Approach}

We considered two different learning settings, each learned on different subsets of the data (Figure 3):

\section{Subject-independent model}

This setting trained a model using the data from all runners except for one. The model was then evaluated on the trials from the one held-aside runner. That is, at training time the model has no access to any data about the runner for whom predictions will be made. As such, this setup estimates the model's accuracy when making predictions for new runners for whom there is no available data, which is interesting in practice. Moreover, the model remains valid if a runner adapts his running style. 


\section{Model Selection, Training and Evaluation}

\section{Subject-dependent model}

For each subject $s_{i}$

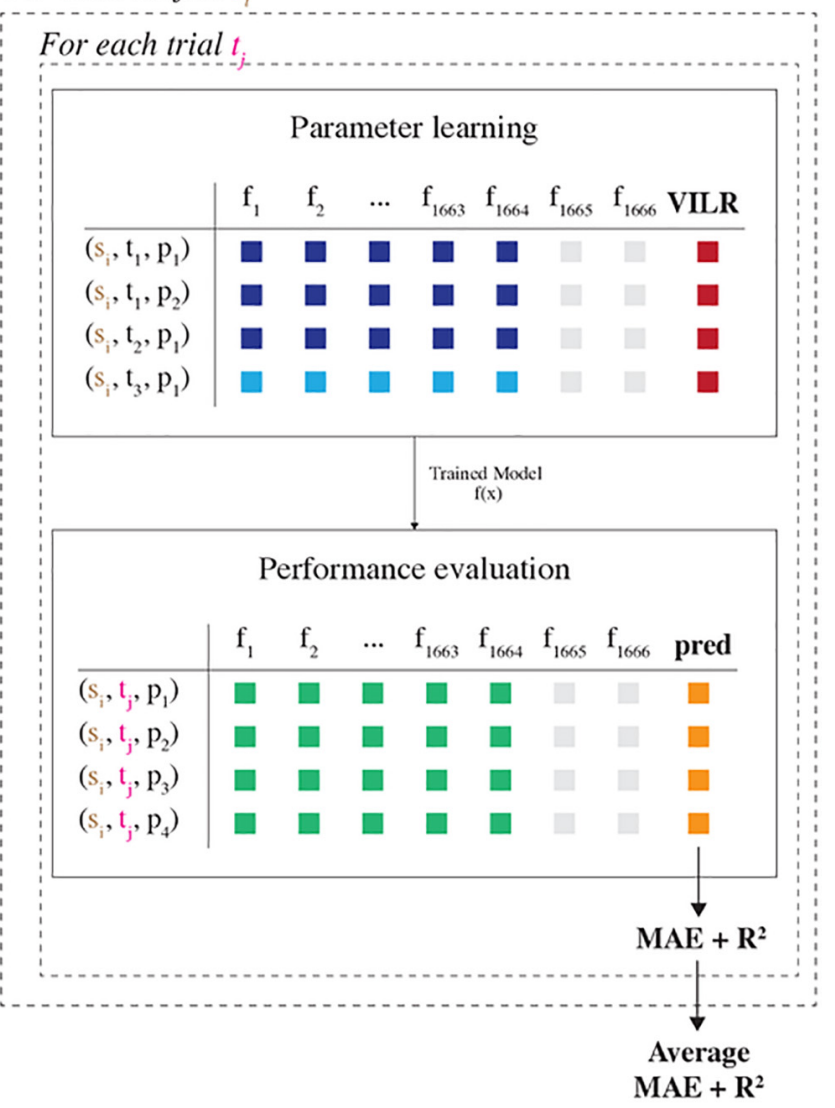

\section{Subject-independent model}

For each subject s

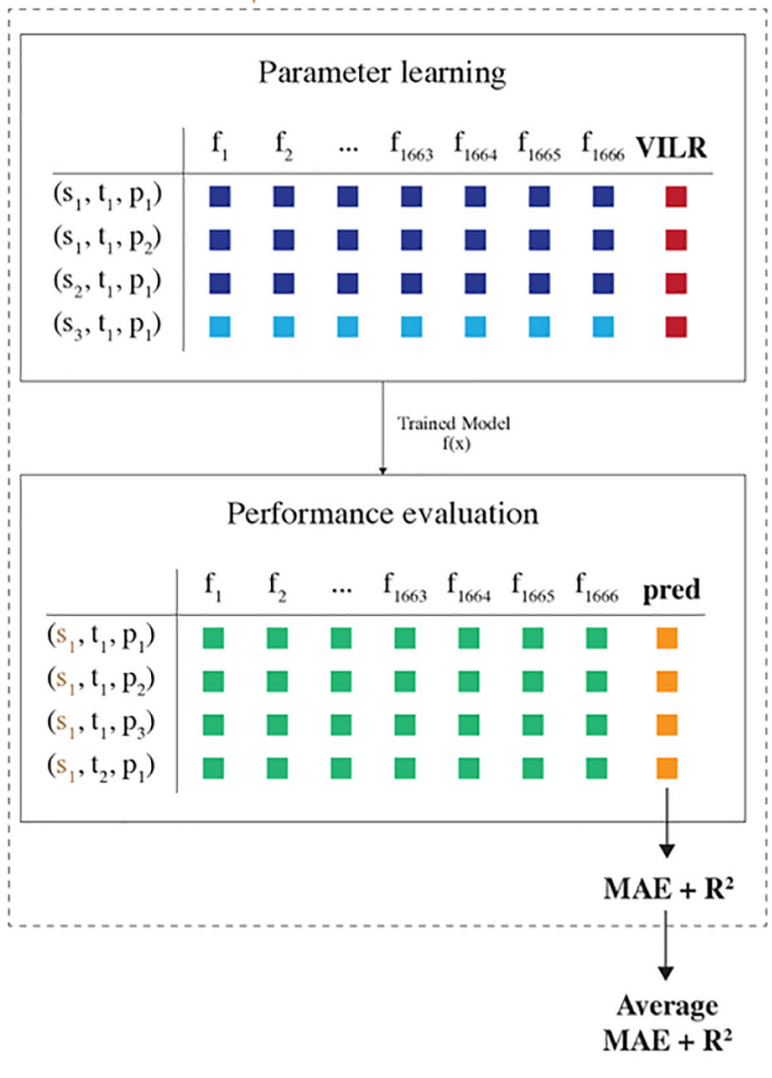

FIGURE 3 | Model selection, training and evaluation part of the machine learning pipeline. Two different learning settings were considered, differing in how the data were split into training and test sets. In the subject-dependent setting, we trained a model on the data of one specific runner, including all trials except one. Subsequently, the model was evaluated on the data of the one held-aside trial. In the subject-independent setting, we trained a model on the data of all runners except for one and evaluated the model on all data of the one held-aside runner. This procedure was repeated for each trial and subject, such that we obtained performance metrics for each fold. Last, the average MAE and $\mathrm{R}^{2}$ score per subject were reported.

\section{Subject-dependent model}

This setting trained a unique personalized model for each subject using only data from that subject. This model would work well if the relationship between the tibial acceleration and the VILR is unique to each subject.

For both settings, we compared the performance of three regression techniques: (1) Linear Regression with Elastic Net regularization (EN), (2) Linear Regression with Least Absolute Shrinkage and Selection Operator regularization (LASSO) and (3) Gradient Boosted Regression Trees (XGB). We used the implementations available in scikit-learn (Pedregosa et al., 2012) for the first two models. For the third regression technique, we used the XGBoost Python package (Chen and Guestrin, 2016).

All models were trained and evaluated in a leave-one-out cross-validation analysis. The subject independent model was iteratively trained on all but one of the subjects to be evaluated on the remaining subject. Similarly, the subject-dependent model is trained on all but one trial of the same subject to be evaluated on the remaining trial. This procedure was repeated for all possible subjects and trials, and the mean accuracy across all folds is reported. As such, this procedure determines the average performance of the models on a group level.

\section{Model Evaluation and Statistical Analysis}

The model's accuracy was assessed using both the mean absolute error (MAE) and the coefficient of determination ( $\mathrm{R}^{2}$ score). The MAE was calculated as the absolute difference between the force platform based VILR and the machine learning predicted VILR. It measures the average magnitude of the errors in the same unit as the VILR and is therefore an easily interpretable measure for the quality of a model. This metric is mainly useful to compare across two models and for domain experts that have insight into the range of VILR values and the magnitude of acceptable errors. The $\mathrm{R}^{2}$ score was computed as $R^{2}=1-\frac{\sum_{i} y_{i}-f_{i}}{\sum_{i} y_{i}-\bar{y}}$, where $y_{i}$ 
are the force plate based VILR values and $f_{i}$ are the machine learning predicted values. It has the advantage of being scalefree, thereby indicating how a model performs compared to a constant baseline.

The number of trials completed by each runner varies substantially. In order to avoid that one runner has an excessively large influence on the accuracy of our models, we computed the global MAE and $\mathrm{R}^{2}$ score in a two-step procedure. First, the average MAE and $R^{2}$ score were calculated over all strides of that runner. Second, the global metrics were then calculated as the average values of these metrics over all runners that completed at least ten trials. This helps prevent the results from being unduly influenced by a single trial or a single runner.

Additionally, we considered two baseline models: a first model that always predicts a runner's average VILR for the corresponding landing foot; and a second linear regression model that only includes the APTA as a covariate.

Repeated measures analysis of variance (ANOVA) was used to examine the effect of various learning settings and regression techniques on the estimated VILR. Post hoc testing was conducted using a Tuckey HSD test on the relative errors. Additionally, Cohen's $d_{r m}$ effect sizes (Lakens, 2013) were computed for the differences in MAE between each machine learning model and the APTA baseline model. We refer to effect sizes as small $(d \leq 0.2)$, medium $(0.2<d \leq 0.8)$ and large $(d>0.8)$ as suggested by Cohen (2013). Statistical analysis was done in Python using the SciPy (ANOVA) and statsmodels (Tuckey HSD) libraries, with the significance level set at $p=0.05$.

To assess the diagnostic ability of each model, it was opted to express the model accuracy in the proportion of correct classifications of high impact runners at a common running speed. Because a cut-off for high impact running at the speed of $3.2 \mathrm{~m} \cdot \mathrm{s}^{-1}$ was lacking, those runners with a mean VILR within the highest $33 \%$ of our database were selected. The diagnostic ability of the models was assessed by calculating their sensitivity and specificity. Sensitivity is the proportion of runners who are correctly categorized as having a high VILR among those who truly have a high VILR. Similarly, specificity is the proportion of runners who are correctly categorized as not having a high VILR among all runners who truly do not have a high VILR. The Receiver Operating Characteristic curves were constructed to demonstrate the trade-off between both metrics using various cut-off values for the predictions.

\section{RESULTS}

\section{Predictive Performance of the Machine Learning Models}

Table 2 summarizes the predictive performance (MAE and $\mathrm{R}^{2}$ scores) of all learned models. In terms of regression techniques, XGB consistently outperformed the other learners $(p<0.05$; in all but the subject-independent model with subject-describing features setting). Therefore, the results of the XGB learner is reported in the remainder of this section. The differences between the different learning settings were all statistically significant
TABLE 2 | Mean absolute error (MAE) $\pm S D$, coefficient of determination $R^{2}$ scores and effect sizes of MAE's versus the axial peak tibial acceleration (APTA) baseline for the estimation of the vertical instantaneous loading rate (VILR) by three different regression models.

\begin{tabular}{|c|c|c|c|c|}
\hline Model & MAE $\left[B W \cdot s^{-1}\right]$ & $\mathbf{R}^{2}$ & $\mathrm{~d}_{r m}$ & Effect size \\
\hline \multicolumn{5}{|c|}{ Subject-independent (without subject-describing features) } \\
\hline APTA & $21.07 \pm 8.13$ & 0.6027 & / & \\
\hline LASSO & $13.13 \pm 8.79$ & 0.7789 & 0.3576 & Medium \\
\hline EN & $12.91 \pm 7.73$ & 0.7811 & 0.3749 & Medium \\
\hline$X G B$ & $12.71 \pm 7.57$ & 0.7397 & 0.4187 & Medium \\
\hline \multicolumn{5}{|c|}{ Subject-independent (with subject-describing features) } \\
\hline APTA & $18.68 \pm 8.44$ & 0.6090 & / & \\
\hline LASSO & $12.75 \pm 9.01$ & 0.7682 & 0.3468 & Medium \\
\hline EN & $12.48 \pm 8.28$ & 0.7713 & 0.3707 & Medium \\
\hline XGB & $12.41 \pm 7.90$ & 0.7741 & 0.4061 & Medium \\
\hline \multicolumn{5}{|c|}{ Subject-dependent } \\
\hline APTA & $7.39 \pm 4.03$ & 0.8500 & l & \\
\hline LASSO & $7.50 \pm 3.45$ & 0.8657 & 0.0168 & Small \\
\hline EN & $7.36 \pm 3.40$ & 0.9124 & 0.0719 & Small \\
\hline$X G B$ & $5.39 \pm 2.04$ & 0.9461 & 0.2900 & Medium \\
\hline
\end{tabular}

Linear Regression with Elastic Net regularization (EN), Linear Regression with Least Absolute Shrinkage and Selection Operator regularization (LASSO), and Gradient Boosted Regression Trees (XGB) in the subject-independent and subjectdependent learning settings.

$(p<0.05)$. A subject-independent model without subjectdescribing features resulted in the least accurate estimations of VILR (MAE: $12.71 \pm 7.57 \mathrm{BW} \cdot \mathrm{s}^{-1} ; \mathrm{R}^{2}$ : 0.7397). Including the subject's weight and shoe type improved the subjectindependent model (MAE: $12.41 \pm 7.90 \mathrm{BW} \cdot \mathrm{s}^{-1} ; \mathrm{R}^{2}: 0.7741$ ). Training a unique model for each subject further improved the predictions by a significant margin (MAE: $5.39 \pm 2.04 \mathrm{BW} \cdot \mathrm{s}^{-1}$; $\left.\mathrm{R}^{2}: 0.9461 ; p<0.01\right)$.

\section{Predictive Performance of the Single Metric Linear Regression Models}

Table 3 shows the predictive performance of linear models that include a single feature in the subject-independent model learning setting. For comparison purposes was the predictive performance of the subject-independent XGB model added as well. Notwithstanding the moderate correlation between the APTA and the VILR, 32 of the extracted features had a higher predictive accuracy than the currently used proxy. Of these 32 features, the mean over the absolute differences between

TABLE 3 | Mean absolute error (MAE) $\pm \mathrm{SD}$ and coefficient of determination $\mathrm{R}^{2}$ scores for the estimation of the VILR by linear regression models using a single variable in the subject-independent model (SIM) learning setting.

\begin{tabular}{lcc}
\hline Statistical model & MAE & $\mathbf{R}^{\mathbf{2}}$ \\
\hline APTA & $21.07 \pm 8.13$ & 0.60 \\
Standard deviation on linear trend & $18.06 \pm 7.28$ & 0.67 \\
Mean over the absolute differences between & $17.47 \pm 7.98$ & 0.71 \\
subsequent acceleration values & & \\
SIM XGB model & $12.41 \pm 7.90$ & 0.77
\end{tabular}


subsequent values of the vertical acceleration signal had the highest correlation with the VILR. A comprehensive overview of all 32 features was made available (Supplementary Table A). The previously discussed regression models that combine multiple of these features still outperform these single-feature models by a large margin.

\section{Diagnostic Ability}

The models' ability to identify runners with a high VILR is shown in Figure 4. With an area under the curve of 0.92, the subjectindependent model XGB had a stronger diagnostic ability than the APTA which has an area under the curve of only 0.82 .

Figure 5 shows cumulatively the percentage of predictions for which the relative error is below a threshold. The subjectindependent model outperformed both baselines by a significant margin. However, the predicted VILR has still an error larger than $25 \%$ for $12 \%$ of the samples in the test set. The subject-dependent fails for only $3 \%$ of the examples.

\section{Computing Time}

The mean calculation time for each prediction was $142 \mathrm{~ms}$ (2.3 GHz Intel Core i5), of which the majority (140 ms) is spent on estimating the ground contact time. Meaning a prediction of the VILR can be made within one foot contact (160 - $350 \mathrm{~ms})$.

\section{DISCUSSION}

The overall aim of this study was to predict the VILR during overground running by creating performant machine learning

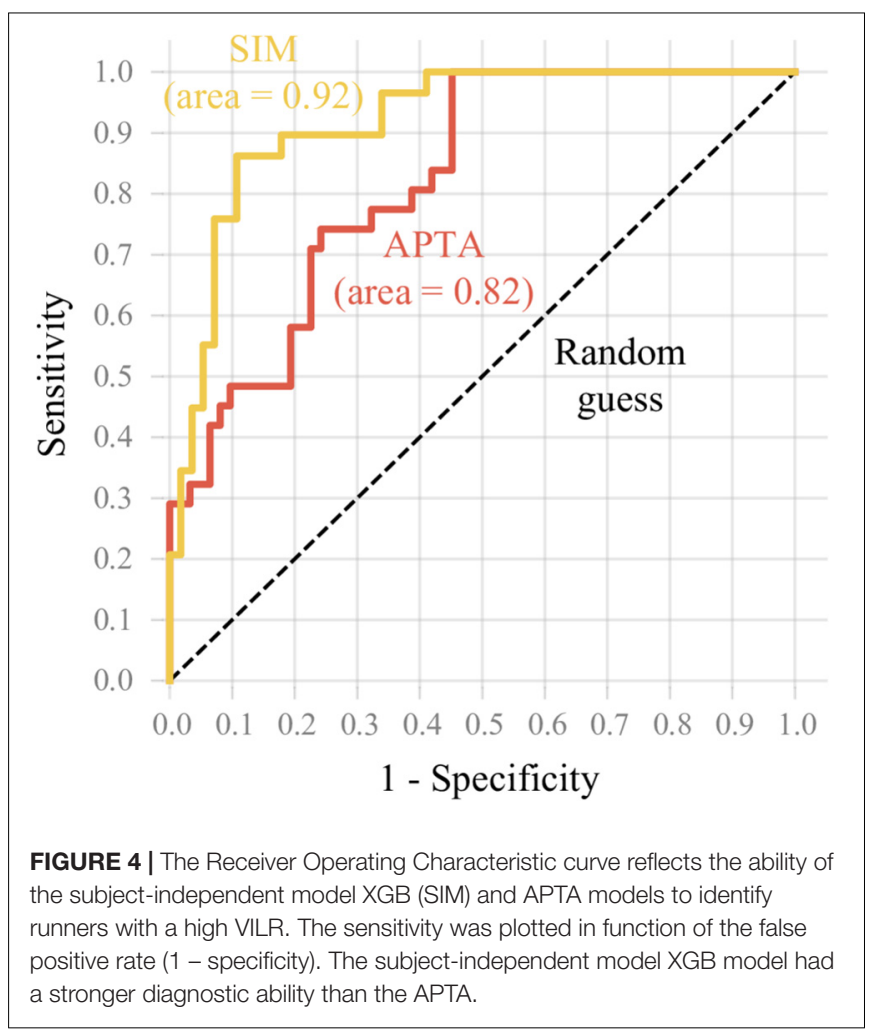

models. Advanced signal processing was used to identify time-discrete features of the 3D acceleration waveforms that discriminate between subtle changes in running biomechanics. Machine-learned models were subsequently built to estimate the VILR and the performance (predictive accuracy, diagnostic ability) of those models were compared to a traditional approach. Two other machine learning techniques not discussed in this study were attempted, but gave unsatisfactory results. First, a data-driven deep recurrent neural network would require much more data than available to learn the complex relations between the tibial acceleration signals and VILR. Second, dynamic time warping was used as a tool for gait-curve matching, incorrectly assuming that runners with similar acceleration profiles have a similar VILR. Moreover, the feature engineering approach is preferable, since the learned models are interpretable (to a certain extend) and have a much lower computational cost.

The findings point out that applying machine learning to multiple 3D tibial acceleration features results in a more accurate prediction of the VILR than the frequently used APTA, which is a single time-discrete variable of tibial acceleration. Additionally, this prediction can be made in real-time, because the data preprocessing (i.e., filtering and feature construction) and prediction requires less calculation time than the typical duration of a single foot contact ( $\sim 250 \mathrm{~ms})$.

Overall, the XGB models systematically outperformed the other learners, suggesting that the XGB model can cope more effectively with the large number of features or that the relationship among the features and target are non-linear (Hepp et al., 2016).

From a machine learning setting perspective, building a subject-dependent model resulted in the most accurate predictions compared to the subject-independent models. The difference in predictive performance between the subjectindependent model and subject-dependent model may partially be explained by the fact that all runners of the second cohort wore their own habitual running footwear, which might influence the measured impact loading. This assumption is further reinforced by the fact that the performance of the subject-independent model can be further improved by incorporating subjectdescribing features (body weight and shoe type). However, the phenotypical variability and choice of footwear can only partly explain the differences in accuracy between a subjectdependent and independent model. Although all runners ran in a similar environment, the ranked order of variable importance for predicting the VILR is unique for each runner in a subject-dependent learned model. Moreover, we observed a large asymmetry between the average VILR for most subject's left and right legs, suggesting that the subject-dependent models could be further improved by building separate models for both legs. However, in our study not mirroring the data resulted in a worse predictive accuracy due to the limited amount of data available for each subject.

The better predictive performance for a subject-dependent model compared to a subject-independent model is in line with previous findings described by Wouda et al. (2018) and Ahamed et al. (2019). However, our subject-independent model is more practical toward real-world applications. It is applicable 


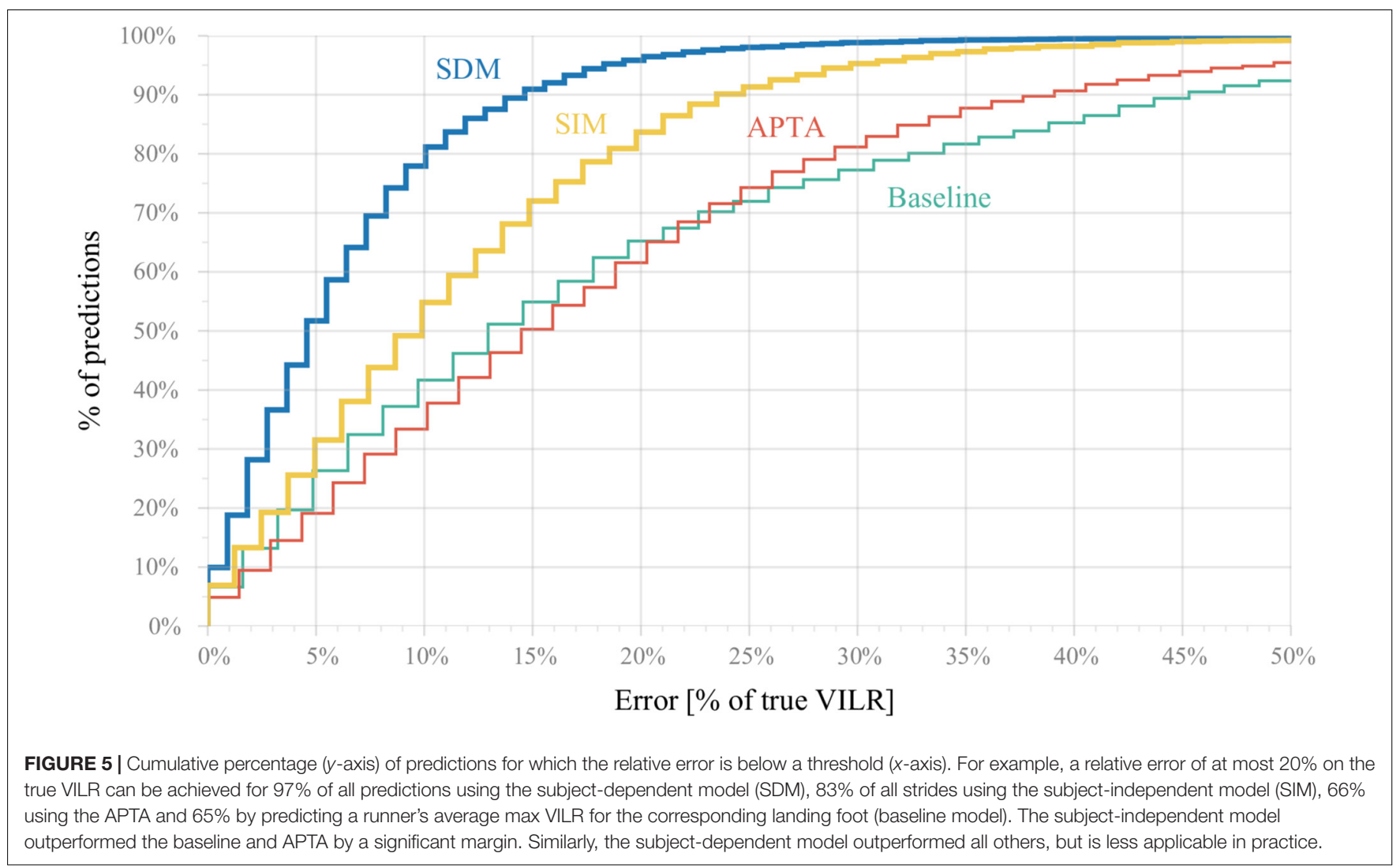

to any runner, regardless of whether prior data is available about the respective runner, which makes this approach generalizable over different subjects. Supporting our hypothesis, the subjectindependent XGB model still outperformed the linear APTA model in terms of prediction accuracy and diagnostic ability.

By incorporating multiple running speeds we were able to create a machine learning algorithm that is capable of making accurate predictions across a broad range of running speeds, making it more usable in practice. As a consequence of this design choice, we observe relatively high $\mathrm{R}^{2}$ scores for these models in comparison with previous research that considered a single running speed (Laughton et al., 2003; Pohl et al., 2009; Greenhalgh et al., 2012; Zhang et al., 2016) due to the restricted range effect (Bland and Altman, 2011): the inclusion of multiple speeds increases the range of the maximal VILR and makes it easier to see the global trend. However, this applies to all models discussed here and therefore does not affect the intermodel differences. For comparison, the evaluation metrics for all models trained on exclusively the most frequent running speed of $3.2 \mathrm{~m} \cdot \mathrm{s}^{-1}$ are provided as Supplementary Table B.

The VILR was predicted accurately, using a broad range of variables derived from filtered $3 \mathrm{D}$ accelerations. In order to screen runners on their VILR at a common training speed of $3.2 \mathrm{~m} \cdot \mathrm{s}^{-1}$ (e.g., identifying runners with a high VILR, during a simple overground running test without the need of an expensive force plate) the classification of runners on impact intensity is preferably done by estimating VILR by means of a machine-learned model instead of relying on the APTA only.
Because VILR is the maximum increase in acceleration of the lower extremity and of the rest of the body during stance (Clark et al., 2017), the predictive accuracy may be further improved by adding trunk acceleration to the accelerometerderived input data.

This study has several limitations. Firstly, we trained the models only on habitual rearfoot strikers. Since machine learning can only be used to memorize patterns that are present in the training data, the trained models can only be applied to other rearfoot strikers and our findings do not necessarily generalize to other foot strike patterns. Secondly, all data was recorded in a laboratory environment. Previous research identified significant variations in APTA or contact time among different running surfaces (Tessutti et al., 2012; Boey et al., 2017). Hence, the findings should be transferred with caution to running on other surfaces.

\section{CONCLUSION}

This study proposes an advanced method to predict VILR during overground running by using only tri-axial shin mounted accelerometers derived data and an XGB machine learning approach. These algorithms, which incorporate time-continuous variables, are able to predict the VILR more accurately than currently possible using a time-discrete variable (e.g., APTA). Since these algorithms do not require significant computational power, they could be implemented on wearables worn by the 
runner in order to screen, monitor or provide biofeedback on the predicted VILR whilst running overground.

\section{DATA AVAILABILITY STATEMENT}

The datasets generated for this study are available upon request to the corresponding authors.

\section{ETHICS STATEMENT}

The studies involving human participants were reviewed and approved by the Ghent University Hospital Ethical Committee. The participants provided their written informed consent to participate in this study.

\section{AUTHOR CONTRIBUTIONS}

RD, PR, PB, DC, VS, and JD conceived, designed, and coordinated the study. RD, JG, and PB collected original data. $\mathrm{PR}$ and $\mathrm{JD}$ developed the machine learning algorithms. RD, PR, PB, and JG participated in data analysis. $\mathrm{RD}$ and $\mathrm{PR}$ developed the figures, initially drafted the manuscript, and the other authors provided useful suggestions in the preparation of the final manuscript.

\section{REFERENCES}

Ahamed, N. U., Kobsar, D., Benson, L. C., Clermont, C. A., Osis, S. T., and Ferber, R. (2019). Subject-specific and group-based running pattern classification using a single wearable sensor. J. Biomech. 84, 227-233. doi: 10.1016/j.jbiomech.2019. 01.001

Bland, J. M., and Altman, D. G. (2011). Correlation in restricted ranges of data. BMJ 342:d556. doi: 10.1136/bmj.d556

Boey, H., Aeles, J., Schütte, K., and Vanwanseele, B. (2017). The effect of three surface conditions, speed and running experience on vertical acceleration of the tibia during running. Sports Biomech. 16, 166-176. doi: 10.1080/14763141. 2016.1212918

Bredeweg, S. W., Kluitenberg, B., Bessem, B., and Buist, I. (2013). Differences in kinetic variables between injured and noninjured novice runners: a prospective cohort study. J. Sci. Med. Sport 16, 205-210. doi: 10.1016/j.jsams.2012. 08.002

Chen, T., and Guestrin, C. (2016). "XGBoost: a scalable tree boosting system," in Proceedings of the 22nd ACM SIGKDD International Conference on Knowledge Discovery and Data Mining - KDD '16, (San Francisco, CA: ACM Press), 785-794. doi: 10.1145/2939672.2939785

Christ, M., Braun, N., Neuffer, J., and Kempa-Liehr, A. W. (2018). Time series feature extraction on basis of scalable hypothesis tests (tsfresh - A Python package). Neurocomputing 307, 72-77. doi: 10.1016/j.neucom.2018.03.067

Christ, M., Kempa-Liehr, A. W., and Feindt, M. (2017). "Distributed and parallel time series feature extraction for industrial big data applications," in Proceedings of the Asian Machine Learning Conference (ACML) 2016, Workshop on Learning on Big Data (WLBD), Hamilton.

Clansey, A. C., Hanlon, M., Wallace, E. S., Nevill, A., and Lake, M. J. (2014). Influence of Tibial shock feedback training on impact loading and running economy. Med. Sci. Sports Exerc. 46, 973-981. doi: 10.1249/MSS. 0000000000000182

Clark, K. P., Ryan, L. J., and Weyand, P. G. (2017). A general relationship links gait mechanics and running ground reaction forces. J. Exp. Biol. 220, 247-258. doi: $10.1242 /$ jeb. 138057
All authors reviewed the manuscript and gave approval for publication.

\section{FUNDING}

This study was funded by the H2020 Interreg EU (Nano4Sports project - 0217), the Research Foundation-Flanders (FWO.3F0.2015.0048.01), the International Society of Biomechanics' matching dissertation grant program 2019, and the KU Leuven Research Fund (C32/17/036).

\section{ACKNOWLEDGMENTS}

We would like to thank Prof. Dr. Benedicte Vanwanseele for her useful suggestions in the preparation of the manuscript. We would also like to thank the participants for their cooperation. Some of these results have been presented at the 2019 Footwear Biomechanics Symposium.

\section{SUPPLEMENTARY MATERIAL}

The Supplementary Material for this article can be found online at: https://www.frontiersin.org/articles/10.3389/fbioe. 2020.00033/full\#supplementary-material

Clermont, C. A., Benson, L. C., Osis, S. T., Kobsar, D., and Ferber, R. (2018). Running patterns for male and female competitive and recreational runners based on accelerometer data. J. Sports Sci. 37, 204-211. doi: 10.1080/02640414. 2018.1488518

Cohen, J. (2013). Statistical Power Analysis for the Behavioral Sciences. Abingdon: Routledge.

Crowell, H. P., and Davis, I. S. (2011). Gait retraining to reduce lower extremity loading in runners. Clin. Biomech. 26, 78-83. doi: 10.1016/j.clinbiomech.2010. 09.003

Falbriard, M., Meyer, F., Mariani, B., Millet, G. P., and Aminian, K. (2018). Accurate estimation of running temporal parameters using foot-worn inertial sensors. Front. Physiol. 9:610. doi: 10.3389/fphys.2018.00610

Fukuchi, R. K., Eskofier, B. M., Duarte, M., and Ferber, R. (2011). Support vector machines for detecting age-related changes in running kinematics. J. Biomech. 44, 540-542. doi: 10.1016/j.jbiomech.2010.09.031

Gerritsen, K. G. M., van den Bogert, A. J., and Nigg, B. M. (1995). Direct dynamics simulation of the impact phase in heel-toe running. J. Biomech. 28, 661-668. doi: 10.1016/0021-9290(94)00127-P

Greenhalgh, A., Sinclair, J., Protheroe, L., and Chockalingam, N. (2012). Predicting impact shock magnitude: which ground reaction force variable should we use? ISSN Int. J. Sports Sci. Eng. 06, 1750-9823.

Hepp, T., Schmid, M., Gefeller, O., Waldmann, E., and Mayr, A. (2016). Approaches to regularized regression - a comparison between gradient boosting and the lasso. Methods Inf. Med. 55, 422-430. doi: 10.3414/ME16-010033

Holzreiter, S. H., and Köhle, M. E. (1993). Assessment of gait patterns using neural networks. J. Biomech. 26, 645-651. doi: 10.1016/0021-9290(93)90028-D

Janssen, D., Schöllhorn, W. I., Newell, K. M., Jäger, J. M., Rost, F., and Vehof, K. (2011). Diagnosing fatigue in gait patterns by support vector machines and self-organizing maps. Hum. Mov. Sci. 30, 966-975. doi: 10.1016/j.humov.2010. 08.010

Kulmala, J.-P., Kosonen, J., Nurminen, J., and Avela, J. (2018). Running in highly cushioned shoes increases leg stiffness and amplifies impact loading. Sci. Rep. 8:17496. doi: 10.1038/s41598-018-35980-35986 
Lakens, D. (2013). Calculating and reporting effect sizes to facilitate cumulative science: a practical primer for t-tests and ANOVAs. Front. Psychol. 4:863. doi: 10.3389/fpsyg.2013.00863

Laughton, C. A., Davis, I. M., and Hamill, J. (2003). Effect of strike pattern and orthotic intervention on tibial shock during running. J. Appl. Biomech. 19, 153-168. doi: 10.1123/jab.19.2.153

Napier, C., MacLean, C. L., Maurer, J., Taunton, J. E., and Hunt, M. A. (2018). Kinetic risk factors of running-related injuries in female recreational runners. Scand. J. Med. Sci. Sports 28, 2164-2172. doi: 10.1111/sms.13228

Ngoh, K. J.-H., Gouwanda, D., Gopalai, A. A., and Chong, Y. Z. (2018). Estimation of vertical ground reaction force during running using neural network model and uniaxial accelerometer. J. Biomech. 76, 269-273. doi: 10.1016/j.jbiomech. 2018.06.006

Op De Beéck, T., Meert, W., Schütte, K., Vanwanseele, B., and Davis, J. (2018). "Fatigue prediction in outdoor runners via machine learning and sensor fusion," in Proceedings of the 24th ACM SIGKDD International Conference on Knowledge Discovery \& Data Mining - KDD '18, (London: ACM Press), 606-615. doi: $10.1145 / 3219819.3219864$

Pedregosa, F., Varoquaux, G., Gramfort, A., Michel, V., Thirion, B., Grisel, O., et al. (2012). Scikit-learn: machine learning in python. J. Machine Learn. Res. 12, 2825-2830.

Pohl, M. B., Hamill, J., and Davis, I. S. (2009). Biomechanical and anatomic factors associated with a history of plantar fasciitis in female runners. Clin. J. Sport Med. Off. J. Can. Acad. Sport Med. 19, 372-376. doi: 10.1097/JSM.0b013e3181b8c270

Preece, S. J., Bramah, C., and Mason, D. (2019). The biomechanical characteristics of high-performance endurance running. Eur. J. Sport Sci. 19, 784-792. doi: 10.1080/17461391.2018.1554707

Robberechts, P., Derie, R., den Berghe, P. V., Gerlo, J., De Clercq, D., Segers, V., et al. (2019). Gait event detection in tibial acceleration profiles: a structured learning approach. arXiv [Preprint] Available at: http://arxiv.org/abs/1910. 13372 (accessed October 30, 2019).

Sheerin, K. R., Reid, D., and Besier, T. F. (2019). The measurement of tibial acceleration in runners-A review of the factors that can affect tibial acceleration during running and evidence-based guidelines for its use. Gait Posture 67, 12-24. doi: 10.1016/j.gaitpost.2018.09.017

Tessutti, V., Ribeiro, A. P., Trombini-Souza, F., and Sacco, I. C. N. (2012). Attenuation of foot pressure during running on four different surfaces: asphalt, concrete, rubber, and natural grass. J. Sports Sci. 30, 1545-1550. doi: 10.1080/ 02640414.2012 .713975

Ueda, T., Hobara, H., Kobayashi, Y., Heldoorn, T. A., Mochimaru, M., and Mizoguchi, H. (2016). Comparison of 3 methods for computing loading rate during running. Int. J. Sports Med. 37, 1087-1090. doi: 10.1055/s-0042-107248
Van den Berghe, P., Six, J., Gerlo, J., Leman, M., and De Clercq, D. (2019). Validity and reliability of peak tibial accelerations as real-time measure of impact loading during over-ground rearfoot running at different speeds. J. Biomech. 86, 238-242. doi: 10.1016/j.jbiomech.2019.01.039

Van Der Worp, H., Vrielink, J. W., and Bredeweg, S. W. (2016). Do runners who suffer injuries have higher vertical ground reaction forces than those who remain injury-free? A systematic review and meta-analysis. Br. J. Sports Med. 50, 450-457. doi: 10.1136/bjsports-2015-094924

van Hees, V. T., Gorzelniak, L., Dean León, E. C., Eder, M., Pias, M., Taherian, S., et al. (2013). Separating movement and gravity components in an acceleration signal and implications for the assessment of human daily physical activity. PLoS One 8:e61691. doi: 10.1371/journal.pone.0061691

Williams, G., Lai, D., Schache, A., and Morris, M. E. (2015). Classification of gait disorders following traumatic brain injury. J. Head Trauma Rehabil. 30, E13-E23. doi: 10.1097/HTR.0000000000000038

Willy, R. W., Buchenic, L., Rogacki, K., Ackerman, J., Schmidt, A., and Willson, J. D. (2016). In-field gait retraining and mobile monitoring to address running biomechanics associated with tibial stress fracture: in-field gait retraining and monitoring. Scand. J. Med. Sci. Sports 26, 197-205. doi: 10.1111/sms.12413

Wouda, F. J., Giuberti, M., Bellusci, G., Maartens, E., Reenalda, J., van Beijnum, B.J. F., et al. (2018). Estimation of vertical ground reaction forces and sagittal knee kinematics during running using three inertial sensors. Front. Physiol. 9:218. doi: $10.3389 /$ fphys.2018.00218

Zeng, W., Liu, F., Wang, Q., Wang, Y., Ma, L., and Zhang, Y. (2016). Parkinson's disease classification using gait analysis via deterministic learning. Neurosci. Lett. 633, 268-278. doi: 10.1016/j.neulet.2016.09.043

Zhang, J. H., An, W. W., Au, I. P. H., Chen, T. L., and Cheung, R. T. H. (2016). Comparison of the correlations between impact loading rates and peak accelerations measured at two different body sites: intra- and inter-subject analysis. Gait Posture 46, 53-56. doi: 10.1016/j.gaitpost.2016.02.002

Conflict of Interest: The authors declare that the research was conducted in the absence of any commercial or financial relationships that could be construed as a potential conflict of interest.

Copyright (c) 2020 Derie, Robberechts, Van den Berghe, Gerlo, De Clercq, Segers and Davis. This is an open-access article distributed under the terms of the Creative Commons Attribution License (CC BY). The use, distribution or reproduction in other forums is permitted, provided the original author(s) and the copyright owner(s) are credited and that the original publication in this journal is cited, in accordance with accepted academic practice. No use, distribution or reproduction is permitted which does not comply with these terms. 